\title{
Successful rituximab treatment for severe rapidly progressive interstitial lung disease with anti-MDA5 antibody-positive juvenile dermatomyositis: A case report and literature review
}

Kentaro Nishi ( $\nabla$ nishi-k@ncchd.go.jp )

National Center for Child Health and Development https://orcid.org/0000-0002-3436-0392

Masao Ogura

National Center for Child Health and Development

Naotaka Tamai

National Center for Child Health and Development

Naofumi Gima

National center for Child Health and Development

Kentaro Ide

National Center for Child Health and Development

Goro Koinuma

National Center for Child Health and Development

Koichi Kamei

National Center for Child Health and Development

Shuichi Ito

Yokohama City University Graduate School of Medicine https://orcid.org/0000-0002-5242-8987

\section{Research Article}

Keywords: dermatomyositis, Anti-melanoma differentiation-association gene 5 antibody, extracorporeal membrane oxygenation, plasma exchange, prone position, children

Posted Date: February 7th, 2022

DOI: https://doi.org/10.21203/rs.3.rs-1322720/v1

License: (1) This work is licensed under a Creative Commons Attribution 4.0 International License.

Read Full License 


\section{Abstract}

Background: Rapidly progressive interstitial lung disease (RP-ILD) is a life-threatening complication of juvenile dermatomyositis (JDM); however, it is generally refractory to treatment and no evidence-based treatment has yet been established for RP-ILD. We present the case of a 2-year-old girl with RP-ILD who was resistant to methylprednisolone, cyclosporine A, cyclophosphamide, immunoglobulin, and plasma exchange. Finally, she required extracorporeal membrane oxygenation, but rituximab contributed to the patient's disease remission and survival. In addition, we conducted a literature review of 18 cases of JDM with RP-ILD.

Case presentation: A 2-year-old girl developed malar rash, mild muscle weakness, and weight loss for a few months before admission. She presented with a history of dry cough and dyspnea for few days, followed by rapid respiratory failure. The diagnosis of JDM with RP-ILD was made by physical examination (malar rashes and Gottron's sign) as well as by the finding of myositis on femoral magnetic resonance imaging, the elevation of serum muscle enzymes, positive anti-melanoma differentiationassociation gene 5 antibody (>7,500 index), the elevation of Krebs von den Lungen- $6(3,420 \mathrm{U} / \mathrm{mL})$, and the finding of extensive ground-glass opacities with consolidation in the bilateral lungs on chest highresolution computed tomography. She received combination therapy, including methylprednisolone pulse therapy, followed by oral prednisolone, intravenous cyclosporine A, intravenous cyclophosphamide, and intravenous immunoglobulin. On the $11^{\text {th }}$ day of her hospital stay, she was put on ventilation, and plasma exchange was started. However, her respiratory condition continued to deteriorate and veno-venous extracorporeal membrane oxygenation was started on day 24 of hospitalization. Rituximab was administered on day 28 of hospitalization. Two weeks after starting rituximab therapy, her respiratory condition gradually started to improve. Eventually, on day 52 of her hospital stay, she was able to wean off extracorporeal membrane oxygenation. Finally, 11 months after admission, she was discharged with minimal ventilatory support and no neurological complications.

Conclusions: According to this literature review, JDM with RP-ILD has a high mortality rate. In JDM, rituximab could be a promising treatment option for RP-ILD. In the future, the effectiveness of rituximab in the early phases of ILD should be investigated.

\section{Background}

Juvenile dermatomyositis (JDM) is a rare autoimmune disease in children ${ }^{1}$, and this is complicated by interstitial lung disease (ILD) in $8-53 \%$ of cases in Japan ${ }^{2,3}$. In particular, rapidly progressive interstitial lung disease (RP-ILD) is a rare but life-threatening complication. JDM-associated RP-ILD is generally refractory to treatment, and no evidence-based treatment has been established. Despite potent combination therapy including methylprednisolone pulse therapy (MPT), calcineurin inhibitor, and/or intravenous cyclophosphamide (IVCY), as well as intravenous immunoglobulin (IVIG), the prognosis of JDM-associated RP-ILD remains unfavorable ${ }^{1}$. However, the efficacy of plasma exchange (PE) or 
rituximab (RTX) has been reported in a small number of DM-associated RP-ILD in adult patients with DM, but the reports in children are limited ${ }^{4-6}$.

Anti-melanoma differentiation-association gene 5 (MDA5) antibody has a significant correlation to JDMassociated ILD ${ }^{4}$. Particularly, JDM patients with a high titer of anti-MDA 5 antibody are likely to develop RP-ILD ${ }^{3,7}$. Here, we report the case of a 2-year-old girl with RP-ILD with anti-MDA5 antibody-positive JDM who was resistant to MPT, cyclosporine A (CyA), IVCY, IVIG, and PE. She finally required veno-venous extracorporeal membrane oxygenation (VV-ECMO), but it was RTX therapy that allowed her to achieve remission and survive.

\section{Case Presentation}

A previously healthy 2-year-old girl developed malar rash, mild muscle weakness, and weight loss for a few months before admission. Her medication history and family history were unremarkable. She was previously admitted to another hospital due to dry cough and dyspnea, which developed a few days ago. She was diagnosed with JDM based on the elevation of serum muscle enzymes and the finding of myositis on femoral magnetic resonance imaging. Her percutaneous oxygen saturation was $96 \%$ on oxygen via nasal canula at $2 \mathrm{~L} / \mathrm{min}$. Six days after diagnosis, her respiratory state rapidly deteriorated and she was transferred to our intensive care unit with a diagnosis of JDM-associated RP-ILD.

Upon admission at our institution, she had low-grade fever $\left(37.4^{\circ} \mathrm{C}\right)$, tachycardia (158 beats per min), tachypnea (56 breaths per min), with an oxygen saturation of $86-96 \%$ on oxygen mask at $8 \mathrm{~L} / \mathrm{min}$. Her height and body weight were $92 \mathrm{~cm}$ and $11.2 \mathrm{~kg}$ with $1.8 \mathrm{~kg}$ loss in 5 months, respectively. Malar rashes and Gottron's sign (i.e., papules on knuckles and elbows) were noted. The laboratory results were as follows: creatinine kinase: $22 \mathrm{U} / \mathrm{I}$, alanine transaminase: $69 \mathrm{U} / \mathrm{I}$, aspartate transaminase: $49 \mathrm{U} / \mathrm{I}$, lactate dehydrogenase: $576 \mathrm{U} / \mathrm{L}$, aldolase: $13.5 \mathrm{U} / \mathrm{L}$ (normal: <6.1 U/L), Krebs von den Lungen-6: 3,420 U/mL (normal: $<500 \mathrm{U} / \mathrm{mL}$ ), anti-MDA5 antibody: $>7,500$ index (normal: $<500$ index), Interleukin-6: $5.2 \mathrm{pg} / \mathrm{mL}$ (normal: $<6 \mathrm{pg} / \mathrm{mL}$ ), and Interleukin-18: $1102.6 \mathrm{pg} / \mathrm{mL}$ (normal: $<500 \mathrm{pg} / \mathrm{mL}$ ). Other autoantibodies and infection-related tests were all negative. The results of the arterial blood gas analysis were as follows: $\mathrm{pH}$ : 7.43, $\mathrm{PaCO}_{2}: 34.1 \mathrm{mmHg}, \mathrm{PaO}_{2}: 68.7 \mathrm{mmHg}$, and $\mathrm{HCO}_{3}{ }^{-}: 22.4 \mathrm{mmol} / \mathrm{L}$ on oxygen mask at $8 \mathrm{~L} / \mathrm{min}$. Chest high-resolution computed tomography (CT) revealed extensive ground-glass opacities with consolidation in the bilateral lungs, greater at the left; these findings are consistent with a diffuse alveolar damage pattern (Fig. I a, b).

Due to the rapid progression of her respiratory failure, the patient was given combination therapy including 4 courses of MPT ( $30 \mathrm{mg} / \mathrm{kg} /$ day for 3 days per course) followed by oral prednisolone (PSL; 1 $\mathrm{mg} / \mathrm{kg} /$ day), intravenous CyA (starting with $3 \mathrm{mg} / \mathrm{kg} /$ day and adjusted to maintain trough levels at 150$200 \mathrm{ng} / \mathrm{mL}), 2$ courses of IVCY (500 mg/m²/dose at a 1 -month interval), and IVIG ( $2 \mathrm{~g} / \mathrm{kg} / \mathrm{dose})$ (Fig. 2 , online). She also received trimethoprim-sulfadiazine as prophylaxis for opportunistic infections. Her respiratory condition deteriorated further, and she was placed on a ventilator support at the 11th hospital day, with the following settings: mean airway pressure (MAP): $12 \mathrm{cmH}_{2} \mathrm{O}$, peak inspiratory pressure (PIP): 
$20 \mathrm{cmH}_{2} \mathrm{O}$, positive end-expiratory pressure (PEEP): $6 \mathrm{cmH}_{2} \mathrm{O}$, and $\mathrm{FiO}_{2}: 0.8$ (oxygen index: 8.0, $\mathrm{PaO}_{2} / \mathrm{FiO}_{2}:$ 150).

However, her respiratory condition worsened further, and her anti-MDA-5 antibodies did not decrease. Thus, we attempted PE on the 11th hospital day (Fig. 2, online), and 23 sessions of PE were performed in total. Serum anti-MDA5 antibody titer then decreased temporarily, but soon rebounded markedly. Furthermore, her respiratory status, including oxygen demand, did not improve at all.

On the 24th hospital day, she could no longer maintain an $\mathrm{SpO}_{2}$ of $90 \%$ at the following ventilator settings: MAP: $18 \mathrm{cmH}_{2} \mathrm{O}$, PIP: $31 \mathrm{cmH}_{2} \mathrm{O}$, PEEP: $10 \mathrm{cmH}_{2} \mathrm{O}, \mathrm{FiO}_{2}: 1.0$ (oxygen index: 20, $\mathrm{PaO}_{2} / \mathrm{FiO}_{2}: 50$ ), and nitric oxide: $5.0 \mathrm{ppm}$.

We decided to introduce VV-ECMO to her for life support. As her respiratory condition was serious, we could not take a follow-up chest CT after her admission. Plain chest radiography at that time showed diffuse ground-glass opacities (Fig. 1C).

After receiving institutional ethical approval, $375 \mathrm{mg} / \mathrm{m}^{2}$ of RTX was given on the 28th hospital day. Mycophenolate mofetil (MMF) was added on the 40th hospital day. Respiratory physical therapy, including being placed in the prone position, was continued under VV-ECMO. Two weeks after starting RTX therapy, her respiratory status gradually started to improve. She was eventually able to wean off ECMO on the 52nd hospital day. Chest CT taken on the 78th hospital day showed improvement of aeration and decreased the consolidation. However, the ground-glass opacities enlarged and traction bronchiectasis was also marked (Fig. 1D-E). Anti-MDA-5 antibody levels also decreased steadily after the first course of RTX, and thus additional RTX was administered at 2 and 6 months due to the recovery of B cells in peripheral blood.

No severe respiratory complications, such as pneumonia, pneumothorax, pulmonary hemorrhage, or thrombosis occurred in the acute phase. We avoided high ventilation pressures above PIP $30 \mathrm{cmH}_{2} \mathrm{O}$ as much as possible to avoid pneumothorax.

Three months after admission, her general condition improved without neurological complications. Her activities of daily living have improved after continuous rehabilitation. She regained the ability to speak, eat, walk, and bathe at $3,4,6$, and 10 months after admission, respectively. Finally, she was discharged with minimum ventilator support at 11 months after admission. At discharge, she could be weaned from the ventilator for short periods of time. The ventilator settings were as follows: MAP: $8 \mathrm{cmH}_{2} \mathrm{O}$, PIP: 14 $\mathrm{cmH}_{2} \mathrm{O}$, PEEP: $7 \mathrm{cmH}_{2} \mathrm{O}$, and $\mathrm{FiO}_{2}: 0.24$. Her chest CT showed remarkable improvement in both lungs at this time as well (Fig. 1F-G).Her anti-MDA5 antibody level was 450 index, and her KL-6 was 1,150 U/m. She is now maintained with PSL $4 \mathrm{mg} /$ day, tacrolimus adjusted with trough levels around $5.0 \mathrm{ng} / \mathrm{mL}$, and MMF $500 \mathrm{mg} /$ day.

\section{Discussion And Conclusions}


Herein, we report the effectiveness of combined therapy including RTX for a 2-year-old girl with lifethreatening RP-ILD associated with anti-MDA5 antibody-positive JDM. Although her condition deteriorated, eventually requiring VV-ECMO, she was rescued with the aforementioned therapies. This case suggests that RTX is an effective option for severe and refractory RP-ILD in JDM.

Anti-MDA-5 antibody-positive JDM and adult DM are known to be more common in Asians than in other races $^{8}$. Notably, in Japan, $28 \%$ of children with JDM and juvenile polymyositis and $10-48 \%$ of adults with DM and polymyositis are positive for anti-MDA- 5 antibodies ${ }^{8}$. Furthermore, cases of anti-MDA- 5 antibodypositive JDM and adult DM are prone to developing RP-ILD, ${ }^{3,7}$ and in JDM specifically, it has a poor prognosis with no established treatment. To further study this, we conducted a literature review of JDM with RP-ILD and found 18 cases (Table 1) ) $^{3,6,8-19}$.

Among 18 patients ( 6 males and 12 females), the median age at disease onset was 6.5 years (range, 216 years). There were 16 Asians, including 14 Japanese patients. Although 11 patients demonstrated apparent muscle weakness, 4 were classified as having juvenile clinically amyopathic dermatomyositis, while the other 3 had no available data. The median months of interval between the onset of JDM and diagnosis of ILD was 3 months (range, 1-8 months). The median months of interval between the diagnosis of JDM and ILD was 1 month (range, 0-7 months). Upon the diagnosis of ILD, respiratory symptoms were observed in 11 patients, while 3 patients were asymptomatic. Furthermore, 12 patients were positive for anti-MDA5 antibody, 1 patient was positive for anti-Jo-1 antibody, and 5 patients were not tested for these. Two patients, including the present case, required ECMO. There were 12 mortalities despite potent combination therapy with MPT, CyA, and/or, IVCY. The duration from the onset of ILD to death was within 4 months in all patients.

Yeung et al. recently reported a case of RP-ILD with JDM treated with multiple agents, including RTX ${ }^{6}$. However, their case had much milder respiratory symptoms than our case. Furthermore, since multiple agents were combined with RTX, it is difficult to evaluate the efficacy of RTX alone. However, the efficacy of RTX has been reported in several adult cases with $\mathrm{DM}^{5}$. Although the mortality of DM-associated RPILD was reported to be about $50 \%$ within 6 months ${ }^{20}$, its prognosis has improved due to early diagnosis and aggressive treatment, including combination therapy with/without RTX ${ }^{5,21}$. In the present case, combination therapy, IVIG, and PE was insufficient to manage RP-ILD, but the patient gradually improved after RTX therapy. RTX also stabilized the serum titer of anti-MDA- 5 antibody. Thus, RTX could be promptly considered in children with RP-ILD refractory to the aforementioned treatments.

There have been a few reports of DM/JDM-associated RP-ILD who were treated with PE, but resulted in poor outcomes ${ }^{4,12,22}$. Some experts suggest that PE might be considered in patients who are unresponsive to combination therapy with corticosteroids and immunosuppressive agents ${ }^{4}$. In our case, the serum titer of anti-MDA5 antibody decreased after frequent $\mathrm{PE}$, but it soon rebounded and the patient's respiratory failure worsened. Both RTX and PE have the same effect in terms of reducing 
autoantibodies, but RTX suppresses antibody production more radically and persistently. In addition, RTX has the advantage of suppressing $T$ cell and $B$ cell interactions and increase of regulatory $T$ cells.

The patient in our case was successfully weaned off ECMO 24 days after administering RTX. Similarly, Zulian et al. reported one pediatric case who survived after VV-ECMO ${ }^{10}$. ECMO can be indicated for lifethreatening respiratory failure, but the prognosis for adult patients with RP-ILD requiring ECMO is poor ${ }^{4}$. ECMO is also used in the bridging therapy to lung transplantation in adult patirnts ${ }^{5}$.

We took several precautions in the management of this critical patient. Trimethoprim-sulfadiazine was administered as prophylaxis for infection. Close monitoring for the growth of Pneumocystis carinii, cytomegalovirus, and fungi were performed. Deep sedation was also done for stable respiratory management. We refrained from using high ventilatory pressures and tolerated hypoxemia and hypercapnia to some extent, because high respiratory pressures may cause pneumothorax and pneumoperitoneum, which can result in sudden respiratory and circulatory collapse ${ }^{23}$. Placing the patient in the prone position may have also contributed to the improvement of respiratory status. We also discussed the use of RTX with the medical team because of the multiple reports of adverse events of interstitial pneumonia caused by RTX itself ${ }^{24}$. In this case, we obtained informed consent from the parents and approval from the hospital's ethics committee.

In conclusion, we rescued a pediatric patient with life-threatening RP-ILD caused by JDM, who required VV-ECMO. Initially, she was resistant to multiple therapeutic strategies, including corticosteroids, immunosuppressive agents, and PE. Finally, the addition of RTX improved her respiratory failure and significantly her anti-MDA-5 antibody titer. Although further studies are needed to confirm our results, we found RTX to be a promising option against RP-ILD in JDM. The usability of RTX in the early phase of ILD should also be studied in the future.

\section{Declarations}

\section{Declarations}

\section{Ethics approval and consent to participate:}

Ethics approval was not applicable. Informed consent was obtained from the individual participant included in this report. The use of rituximab was approved by the institutional ethics committee of the National Center for Child Health and Development (approval no. 2020-03).

\section{Consent for publication:}

Consent for publication was obtained from the individual's guardian included in this report. Written informed consent was obtained from the legal guardians as participants were under 16 years of age. This was applicable for all potentially identifiable information contained within the manuscript file and this is available for review by the editor of the journal. 


\section{Declarations}

\section{Competing interests:}

There was no external funding for this manuscript. Koichi Kamei has received research funding from the Terumo Foundation for Life Sciences and Arts, and donations from Ono Pharmaceutical Co., Ltd. Shuichi Ito has received employment from TEIJIN PHARMA Ltd., Honoraria from SANOFI K.K., and research funding from TEIJIN PHARMA Ltd. and Astellas PHARMA Co., Ltd. The other authors have no potential conflicts of interest to disclose.

\section{Declarations}

\section{Author's contributions}

KN prepared the manuscript; MO, NT, and NG edited and reviewed the manuscript; GK and KK revised the manuscript; and SI revised and oversaw the work. All authors participated in the discussions about the manuscript and approved the final version.

\section{Funding:}

There was no external funding for this manuscript.

\section{Acknowledgments}

The authors would like to thank Dr. Satoshi Sato for adding information in reference 12. The authors would also like to thank the medical editors from the Division of Education for Clinical Research at the National Center for Child Health and Development for editing a draft of this manuscript.

\section{Abbreviations}

JDM, Juvenile dermatomyositis; ILD, Interstitial lung disease; RP-ILD, rapidly interstitial lung disease; MDA-5, melanoma differentiation-association gene 5; MPT, methylprednisolone pulse therapy; IVCY, intravenous cyclophosphamide; IVIG, intravenous immunoglobulin; RTX, rituximab; PE, plasma exchange; MMF, mycophenolate mofetil; VV-ECMO, veno-venous extracorporeal membrane oxygenation; PSL, prednisolone; CyA, cyclosporine A.

\section{Declarations}


Ethics approval and consent to participate: Ethics approval was not applicable. Informed consent was obtained from the individual participant included in this report. The use of rituximab was approved by the institutional ethics committee of the National Center for Child Health and Development (approval no. 2020-03).

Consent for publication: Consent for publication was obtained from the individual's guardian included in this report. Written informed consent was obtained from the legal guardians as participants were under 16 years of age. This was applicable for all potentially identifiable information contained within the manuscript file and this is available for review by the editor of the journal.

Availability of data and materials: The datasets used and/or analyzed during the current study are available from the corresponding author upon reasonable request.

Competing interests: There was no external funding for this manuscript. Koichi Kamei has received research funding from the Terumo Foundation for Life Sciences and Arts, and donations from Ono Pharmaceutical Co., Ltd. Shuichi Ito has received employment from TEIJIN PHARMA Ltd., Honoraria from SANOFI K.K., and research funding from TEIJIN PHARMA Ltd. and Astellas PHARMA Co., Ltd. The other authors have no potential conflicts of interest to disclose.

Funding: There was no external funding for this manuscript.

Author's contributions: KN prepared the manuscript; MO, NT, and NG edited and reviewed the manuscript; GK and KK revised the manuscript; and SI revised and oversaw the work. All authors participated in the discussions about the manuscript and approved the final version.

\section{Acknowledgments}

The authors would like to thank Dr. Satoshi Sato for adding information in reference 12. The authors would also like to thank the medical editors from the Division of Education for Clinical Research at the National Center for Child Health and Development for editing a draft of this manuscript.

\section{References}

1. Kobayashi I, Akioka S, Kobayashi N, Iwata N, Takezaki S, Nakaseko H, et al. Clinical practice guidance for juvenile dermatomyositis (JDM) 2018-Update. Mod Rheumatol. 2012;30:411-23.

2. Kishi T, Miyamae T, Hara R, Nakajima S, Imagawa T, Mori M, et al. Clinical analysis of 50 children with juvenile dermatomyositis. Mod Rheumatol. 2013;23:311-7.

3. Kobayashi N, Takezaki S, Kobayashi I, Iwata N, Mori M, Nagai K, et al. Clinical and laboratory features of fatal rapidly progressive interstitial lung disease associated with juvenile dermatomyositis. Rheumatology. 2015;54:784-91.

4. Romero-Bueno F, Diaz Del Campo P, Trallero-Araguás E, Ruiz-Rodríguez JC, Castellvi I, RodriguezNieto MJ, et al. Recommendations for the treatment of anti-melanoma differentiation- associated 
gene 5-positive dermatomyositis-associated rapidly progressive interstitial lung disease. Semin Arthritis Rheum. 2020;50:776-90.

5. Huang K, Vinik O, Shojania K, Yeung J, Shupak R, Nimmo M, et al. Clinical spectrum and therapeutics in Canadian patients with anti-melanoma differentiation-associated gene 5 (MDA5)-positive dermatomyositis: a case-based review. Rheumatol int. 2019;39:1971-81.

6. Yeung TW, Cheong KN, Lau YL, Tse KN. Adolescent-onset anti-MDA5 antibody-positive juvenile dermatomyositis rapidly progressive interstitial lung disease and spontaneous pneumomediastinum: a case report and literature review. Pediatr Rheumatol Online J. 2021;19:103.

7. Kobayashi I, Okura Y, Yamada M, Kawamura N, Kuwana M, Ariga T. Anti-melanoma differentiationassociated gene 5 antibody is a diagnostic and predictive marker for interstitial lung disease associated with juvenile dermatomyositis. J Pediatr. 2011;158:675-7.

8. Ueki M, Kobayashi I, Takezaki S, Tozawa Y, Okura Y, Yamada M, et al. Myositis-specific autoantibodies in Japanese patients with juvenile idiopathic inflammatory myopathies. Mod Rheumatol. 2019;29:351-6.

9. Tosun A, Serdaroğlu G, Aslan MT, Polat M, Akalin T, Tekgul H, et al. Severe juvenile dermatomyositis: two patients complicated with extra musculocutaneous involvement. Rheumatol int. 2006;26:10403.

10. Zulian F, Martinez Toledo MM, Amigoni A, Martini G, Agosto C, et al. Successful use of extracorporeal membrane oxygenation for severe interstitial lung disease in a child with dermatomyositis. Intensive Care Med. 2007;33:1663-6.

11. Nonaka Y, Imanaka K, Nerome Y, Maeno N, Akaike N, Arimura H, et al. A case of anti-Jo-1 antibodypositive juvenile dermatomyositis complicated by interstitial lung disease: successful treatment with cyclosporine A [in Japanese]. J jpn Pediatr Soc. 2008;112:719-23.

12. Nagai $Y$, Mizuno T, Yoshizawa $C$, Ishikawa O. Fatal interstitial pneumonia in juvenile dermatomyositis. Eur J Dermatol. 2010;20:208-10.

13. Sakurai N, Nagai K, Tsutsumi H, Ichimiya S. Anti-CADM-140 antibody-positive juvenile dermatomyositis with rapidly progressive interstitial lung disease and cardiac involvement. J Rheumatol. 2011;38:963-4.

14. Kobayashi I, Okura Y, Yamada M, Kawamura N, Kuwana M, Ariga T. Anti-melanoma differentiationassociated gene 5 antibody is a diagnostic predictive marker for interstitial lung disease associated with juvenile dermatomyositis. J Pediatr. 2011;158:675-7.

15. Kobayashi I, Ono S, Kawamura N, Okano M, Miyazawa K, Shibuya H, et al. KL-6 is a potential marker for intestitial lung disease associated with juvenile dermatomyositis. J Pediatr. 2001;138:274-6.

16. Kobayashi I, Yamada M, Takahashi Y, Kawamura N, Okano M, Sakiyama Y, et al. Interstitial lung disease associated with juvenile dermatomyosistis: clinical features and efficacy of cyclosporin $A$. Rheumatology (Oxford). 2003;42:371-4.

17. Ishikawa J, Shikama Y, Takahashi E, Akagi K. Rapidly progressive interstitial pneumoniae with juvenile dermatomyositis [in Japanese]. J Jpn Pediatr Soc. 2011;115:793-9. 
18. Sato S, Uejima Y, Nanbu M, Suganuma E, Takano T, Tanaka R, et al. Clinical analysis and outcome of interstitial lung disease complicated with juvenile dermatomyositis and juvenile polymyositis. Mod Rheumatol. 2017;27:652-6.

19. Hou J, Zhou ZX, Li JG, Xu YJ, Ding YC. Three cases report of juvenile dermatomyositis with positive anti-melanoma differentiation associated gene 5 (MDA5) antibody and severe interstitial lung disease and literature review. Zhonghua Er Ke Za Zhi. 2019;57:928-33.

20. Ye S, Chen XX, Lu XY, Wu MF, Deng Y, Huang WQ, et al. Adult clinically amyopathic dermatomyositis with rapid progressive interstitial lung disease: a retrospective cohort study. Clin Rheumatol. 2007;26:1647-54.

21. Matsushita T, Mizumaki K, Kano M, Yagi N, Tennichi M, Takeuchi A, et al. Antimelanoma differentiation-associated protein 5 antibody level is a novel tool for monitoring disease activity in rapidly progressive interstitial lung disease with dermatomyositis. Br J Dermatol. 2017;176:395-402.

22. Kagawa H, Tsujino $K$, Yamamoto $Y$, Iwai A, Hara R, Matsuki T, et al. Acute lung injury after plasma exchange in a patient with anti-MDA5 antibody-positive, rapidly progressive, interstitial lung disease: A case report. Respir Med Case Rep. 2020;1:101016.

23. Anzueto A, Frutos-Vivar F, Esteban A, Alía I, Brochard L, Stewart T, et al. Incidence, risk factors and outcome of barotrauma in mechanically ventilated patients. Intensive Care Med. 2004;30:612-9.

24. Wagner SA, Mehta AC, Laber DA. Rituximab-induced interstitial lung disease. Am J Hematol. 2007;82:916-9.

\section{Tables}

Table 1 is only available as a download in the Supplemental Files section.

\section{Figures}


(a)

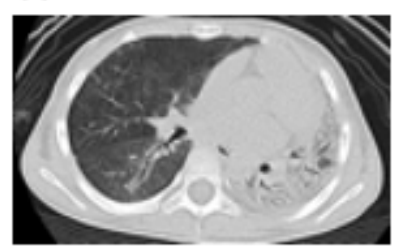

(b)

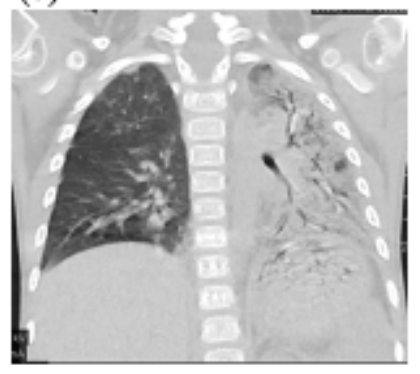

(c)

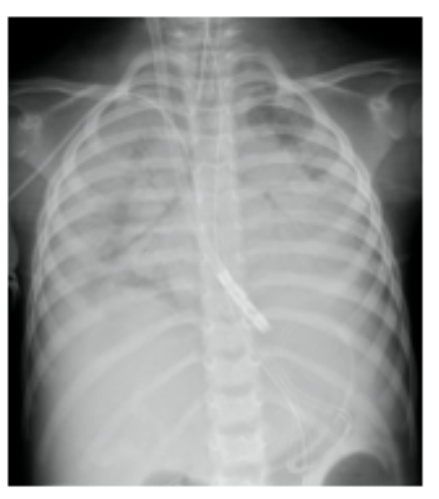

(d)

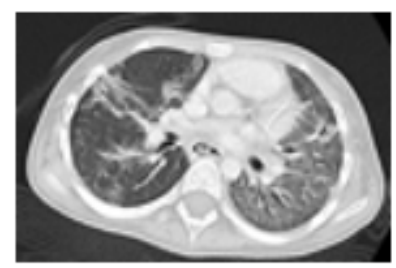

(e)

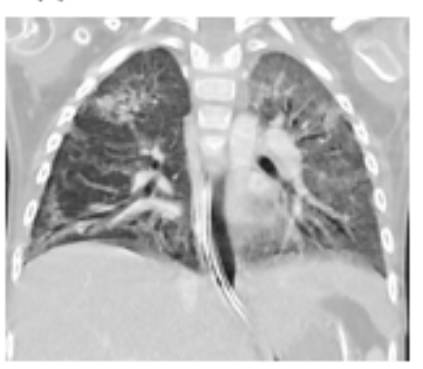

(f)

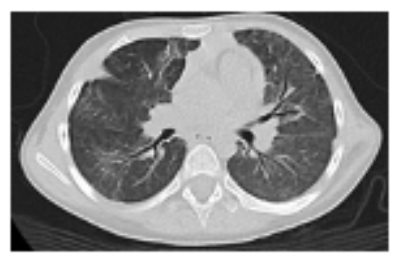

(g)

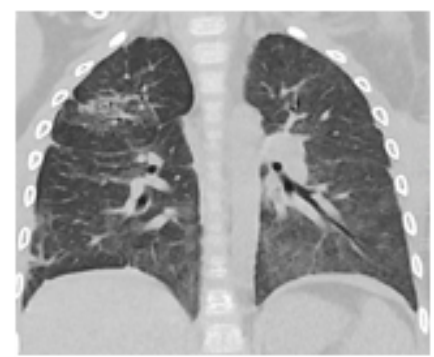

\section{Fig I}

\section{Figure 1}

\section{Chest computed tomography of the patient}

(A, B) Lung computed tomography (CT) scan at admission showing extensive ground-glass opacities with consolidation in bilateral lungs, significant at the left side. (C) Chest X-ray upon initiation of venovenous extracorporeal membrane oxygenation showing massive bilateral areas of consolidation. (D, E) Lung CT scan after treatment on the $78^{\text {th }}$ hospital day showing improvement in aeration and resolution of consolidation. Ground-glass opacities were enlarged and traction bronchiectasis was evident. $(F, G)$ Lung CT scan at 11 months after admission showing improvement in both lungs. 


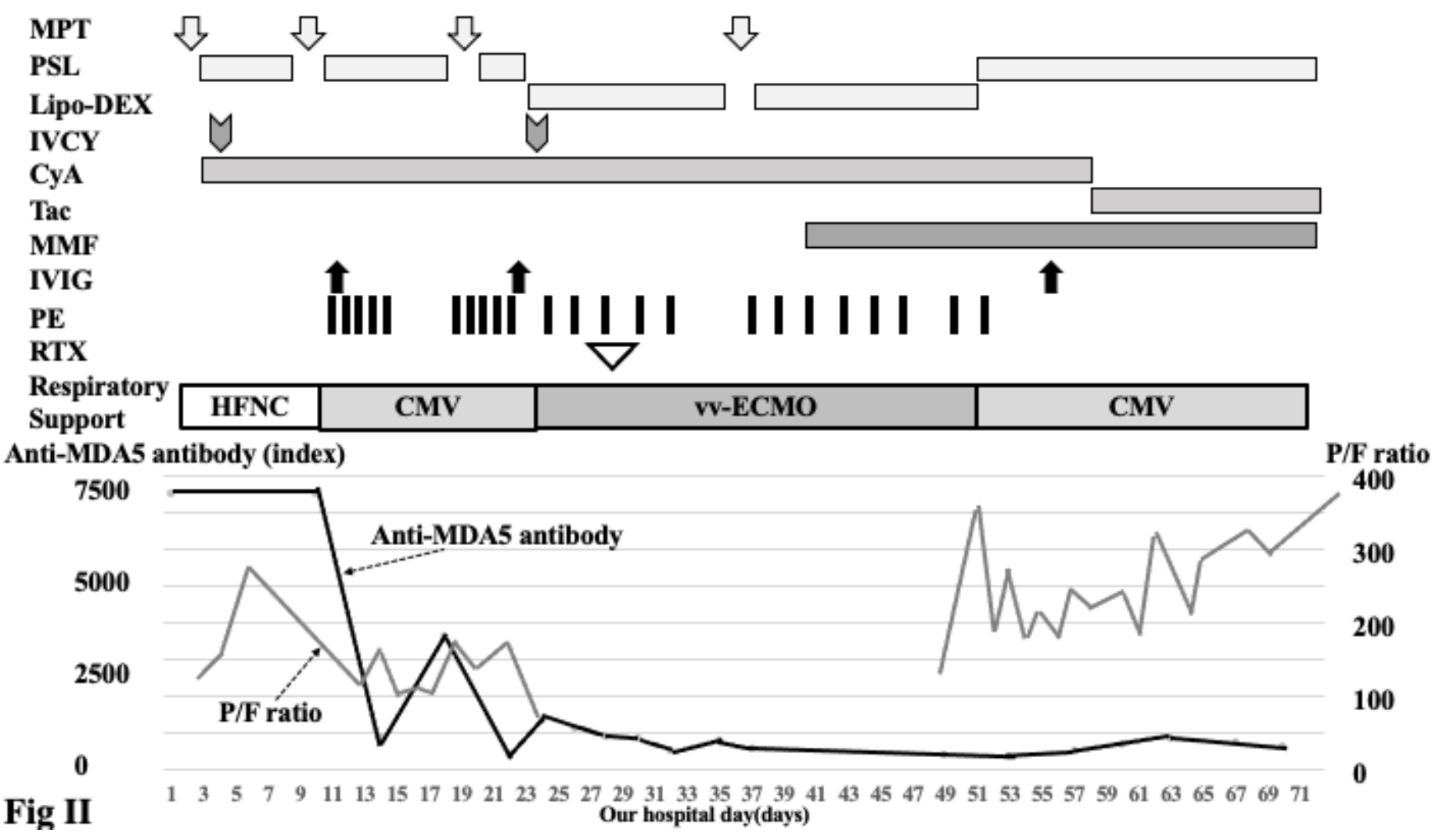

Figure 2

\section{Clinical course and treatment.}

Clinical course and treatment of the patient. MPT, methylprednisolone pulse therapy; PSL, prednisolone; Lipo-Dex, liposteroid dexamethasone; IVCY, intravenous cyclophosphamide; CyA, cyclosporine A; Tac, tacrolimus; MMF, mycophenolate mofetil; IVIG, intravenous immunoglobulin; PE, plasma exchange; RTX, rituximab; HFNC, High-flow nasal cannula; CMV, conventional mechanical ventilation; VV-ECMO, venovenous extracorporeal membrane oxygenation; MDA5, melanoma differentiation-association gene 5; P/F ratio, $\mathrm{PaO} 2 / \mathrm{FiO} 2$ ratio.

\section{Supplementary Files}

This is a list of supplementary files associated with this preprint. Click to download.

- Table1.docx 